\title{
Análise da Influência do Mercado Financeiro sobre o Mercado Futuro Agropecuário no Brasil
}

\author{
Viviane Araujo da Silva ${ }^{1}$ \\ Leonardo Bornacki de Mattos $^{2}$ \\ Alexandre Bragança Coelho ${ }^{3}$
}

Resumo: A trajetória altista dos preços das commodities, iniciada em 2002, tem sido acompanhada pelo aumento do volume de negociações nos mercados futuros. Nesse contexto, a migração de investimentos dos mercados financeiros para os mercados futuros tem sido apontada como um dos fatores responsáveis pelo aumento dos preços e da volatilidade no mercado de alimentos. Isto posto, objetivou-se neste trabalho analisar a influência do mercado financeiro sobre o mercado futuro agropecuário no Brasil. Os resultados mostram ter ocorrido transmissão de volatilidade do mercado financeiro internacional para o mercado futuro de milho, café arábica e soja; e do mercado financeiro nacional para o mercado futuro de milho. Com exceção da relação Ibovespa-mercado futuro de boi gordo, não há relação de causalidade entre a volatilidade dos mercados, assim como não há relação linear causal partindo da variação de volume de contratos futuros negociados em direção aos retornos dos preços.

Palavras-Chave: Mercado futuro, volatilidade, commodities agropecuárias.

Classificação JEL: Q13; Q14. 


\title{
Analysis of the Influence of Financial Market on Brazilian Agricultural Futures Market
}

\begin{abstract}
The upward trend in commodity prices that began in 2002, has been accompanied by increased trading volume in future markets. In this context, the migration of investments from financial markets for the future markets has been identified as one of the factors responsible for the increase in these prices and volatility in the food market. Thus, this study aimed to analyze the influence of financial markets on the agricultural futures markets in Brazil. Results show that there has been transmission of volatility from international financial markets toward corn, coffee and soybeans future markets; and from the domestic financial market toward corn future market. The causality-in-variance test indicates that, except for the Ibovespa-cattle future market relation, there is no evidence of causality between the financial market and the future market volatility. There is no linear causal relationship from the variation in volume of futures contracts traded towards returns of prices.
\end{abstract}

Keywords: Future market, volatility, agricultural commodities.

JEL Classification: Q13; Q14.

\section{Introdução}

Ao longo das últimas décadas, os preços das commodities registraram vários períodos de grande oscilação, tanto com tendência de baixa quanto de alta. O último movimento observado iniciou-se no ano de $2002 \mathrm{e}$, contrariando as expectativas, mostrou-se persistente e abrangente, atingindo tanto as soft commodities, que são os produtos cultivados, quanto as hard commodities, que são os produtos extraídos ou minerados. Com exceção de breves e esporádicos períodos, os preços mantiveram sua trajetória ascendente no decorrer da década.

Várias explicações têm sido apresentadas para justificar o boom dos preços no mercado de commodities, fundamentando-se, principalmente, na existência de uma oferta contraída em face de uma demanda crescente. Esse cenário estaria sendo fomentado, entre outros fatores, pelo crescente aumento da demanda mundial, destacando-se China e Índia, pelo aumento da utilização de cereais para a produção de ração animal e pelo incentivo à produção de biodiesel, enquanto que, atuando do lado da oferta, estariam as mudanças climáticas e a manutenção de baixos estoques.

Outro fator abordado pela literatura, e que se encontra no cerne deste estudo, 
é o aumento da atividade especulativa no mercado futuro de commodities. Defende-se que este mercado estaria recebendo um influxo anormal de dinheiro, aplicado em sua grande parte por especuladores que, pessimistas quanto às aplicações financeiras tradicionais, estariam buscando a melhora da relação risco-retorno de suas carteiras de investimento. Esse processo, caracterizado pelo aumento do fluxo de investimentos vindos dos mercados financeiros tradicionais em direção aos mercados futuros de commodities, tem sido denominado por alguns autores ${ }^{1}$ como "financeirização do mercado de commodities".

Vários estudos (Abanomey \& Mathur 2001; Erb \& Harvey 2006; You \& Daigler 2007; Smimou 2010) comprovam que a diversificação de portfólios pode gerar aos investidores benefícios maiores quando contratos futuros de commodities são adicionados ao conjunto de investimentos de uma carteira, proporcionando maiores retornos e diminuição do risco. Gorton \& Rouwenhorst (2006) analisam o desempenho das commodities como ativos financeiros e verificam que as mesmas apresentam correlação negativa com outros ativos e que, em determinadas situações, como em períodos inflacionários, registram, na média, um desempenho melhor que títulos e ações.

Masters (2008) é enfático ao afirmar que o aumento dos preços das commodities em geral, observado principalmente no período da crise financeira do subprime (2007-2008), é decorrente do crescimento da utilização de commodities como ativos de investimento pelos chamados investidores institucionais. O aumento da participação de investidores que só comercializam em futuros, não tendo qualquer ligação com o mercado físico, estaria provocando o aumento, tanto das cotações futuras, quanto dos preços nos mercados físicos.

Nos EUA, de acordo com Irwin \& Sanders (2011), o investimento em ativos financeiros ligados aos mercados futuros de commodities, incluindo ativos comercializados dentro e fora do país, foi de aproximadamente US\$ 200 bilhões, no final de 2004, alcançando US $\$ 250$ bilhões, em meados de 2008, chegando a US\$ 300 bilhões no segundo semestre de 2010. Domanski \& Heath (2007) ressaltam que, embora esses investimentos sejam pequenos em relação ao mercado financeiro global, é grande em relação à produção no mercado físico.

A tendência de crescimento das negociações nos mercados futuros também pode ser observada no Brasil. Entre os anos de 2000 e 2008, o segmento de derivativos agropecuários da BM\&FBOVESPA registrou crescimento sucessivo no número de contratos negociados, sendo que, em 2008, auge da crise financeira do subprime ${ }^{2}$, o setor registrou recorde de negociações, chegando

1 Domanski \& Heath (2007), Mayer (2009), United Nations Conference on Trade and Development - UNCTAD, em seu Trade and Development Report (2009), Baffes \& Haniotis (2010), Tang \& Xiong (2010), entre outros.

2 Nesta pesquisa, os anos de 2007 e 2008 são considerados como anos de início e fim da crise financeira do subprime, o que não quer dizer que a mesma, necessariamente, tenha iniciado e acabado nesse período.

Trata-se unicamente de uma hipótese metodológica para atender ao objetivo da pesquisa. 
a 3.279.827 contratos transacionados. No pós-crise, apesar de uma queda do volume comercializado, as médias mantiveram-se acima das verificadas no período pré-crise.

Entretanto, não é possível afirmar que o crescimento do volume de comercialização de contratos de commodities seja exclusivamente reflexo do aumento da participação de investidores (oriundos do mercado financeiro) nos mercados futuros. No Brasil, dados disponibilizados pela BM\&FBOVESPA mostram que, no segmento agropecuário, considerando o período 2002-2011, foi pequeno o incremento da participação de investidores institucionais nacionais ${ }^{3}$ e investidores estrangeiros na comercialização de contratos futuros.

Ressalta-se, todavia, que a dificuldade está em classificar as operações realizadas por hedgers daquelas realizadas por investidores sem ligação com a comercialização no mercado físico, ou seja, os especuladores, visto que até aqueles investidores tidos como hedgers podem especular nos mercados futuros. Outro ponto importante a ser considerado é que, ao analisar a alta de preços das commodities agropecuárias, é imprescindível diferenciar a mudança na tendência de preços, referente às alterações estruturais de oferta e demanda, a médio e longo prazo, da volatilidade, referente às mudanças na taxa de variação dos preços em períodos ininterruptos.

Nesta pesquisa, a relação entre o mercado financeiro e o mercado futuro de commodities agropecuárias é abordada sob a perspectiva da volatilidade, uma vez que existe a hipótese de que a ação especulativa de investidores no mercado futuro de alimentos esteja produzindo uma bolha, responsável em grande parte pelo aumento da volatilidade dos preços.

Deste modo, considerando o exposto, objetivou-se, por meio da análise do processo de transmissão de volatilidade entre os mercados financeiros, internacional e nacional, e o mercado futuro agropecuário, assim como pela investigação da existência de causalidade entre as volatilidades desses mercados, estudar a influência do mercado financeiro sobre o mercado futuro agropecuário no Brasil.

Da mesma forma, partindo-se da hipótese de que o aumento do volume de negociações nos mercados futuros esteja causando a elevação dos preços das commodities agropecuárias, verifica-se a existência de relação causal entre o volume de contratos futuros negociados e os preços desses contratos.

Este trabalho é composto por três seções, além desta parte introdutória. A primeira seção apresenta o referencial teórico. A segunda seção é reservada para a apresentação da metodologia. Na terceira seção, são apresentados e discutidos os resultados alcançados. Por fim as conclusões. 


\section{Referencial Teórico}

Nesta seção objetiva-se fundamentar, sob a perspectiva teórica, a migração de capitais dos mercados financeiros, internacional e nacional, para o mercado de commodities agropecuárias, assim como estabelecer relação entre as variações no volume e nos preços dos contratos futuros, dada a chegada de novas informações no ambiente de negociação.

\subsection{A Moderna Teoria do Portfólio}

O investimento financeiro nos mercados futuros de commodities tem se tornado gradativamente mais significativo, revelando a nova dinâmica que se configura no mercado financeiro internacional. $\mathrm{O}$ aumento do fluxo especulativo nas bolsas de mercadorias retrata a nova tendência dos investidores, cujo comportamento está fundado sobre os preceitos da moderna teoria de seleção de carteiras, idealizada por Markowitz (1952).

De acordo com Markowitz, é possível reduzir o risco de uma carteira de investimento sem comprometer seu retorno, combinando-se ativos que apresentam correlação negativa entre seus rendimentos individuais. Sedimentado nesse princípio, Bergstrom (1975) apresenta a teoria da diversificação internacional de portfólio, na qual defende que a inclusão de ativos comercializados em outros países melhora a relação risco-retorno das carteiras de investimento, benefício decorrente do fato de os retornos dos ativos domésticos apresentarem baixa correlação com os ativos estrangeiros.

Ademais, estudos demonstram que a eficácia da diversificação de portfólios pode ser aumentada com a adição de commodities ao rol de investimentos de uma carteira, melhorando a gestão do risco, o que justifica o investimento em contratos futuros negociados em outros países.

Deve-se considerar ainda que, a escolha do portfólio ótimo, que possibilita a maximização do retorno e a minimização do risco, vai acabar por determinar a demanda por ativos específicos e, consequentemente, seu preço. Um dos modelos convencionais mais utilizados na alocação de ativos em carteiras de investimento é o modelo CAPM ${ }^{4}$.

\subsection{Modelo de Negociação de Ativos}

A financeirização tem sido apontada como um dos fatores responsáveis pela

4 O Capital Asset Pricing Model (CAPM) é um modelo derivado da Teoria do Portfólio de Markowitz (1952). Segundo o modelo CAPM, cujos pressupostos básicos foram disseminados pelos trabalhos pioneiros de Sharpe (1964) e Lintner (1965), os investidores tendem a exigir maior retorno quanto maior for o risco do investimento. 
instabilidade dos mercados futuros de alimentos. Segundo essa hipótese, o aumento da atividade especulativa nos mercados futuros de commodities estaria produzindo uma bolha, responsável em grande parte pelo incremento do volume de transações e pela inflação dos preços dos alimentos.

Um dos modelos que aborda a dinâmica envolvida no processo de funcionamento do mercado financeiro, no que tange ao volume transacionado e ao comportamento dos preços, foi desenvolvido por Copeland (1976) que, apesar de não tratar especificamente de mercado futuro, fornece subsídios para a compreensão do comportamento deste.

O modelo considera a entrada sequencial de informações no mercado, assumindo que, antes da chegada dessas informações, todos os participantes apresentam curvas de demanda homogênea.

A partir da incorporação da nova informação pelos traders, ocorre o reequilíbrio do mercado, que se divide em dois estágios: I) todos os participantes do mercado, tantos os informados quanto os não informados, detêm quantidades positivas de ativo e II) todos os participantes desinformados vendem a totalidade de seus ativos aos participantes informados.

O modelo ainda indica a existência da relação entre o volume de ativos negociados e o número de participantes atuantes no mercado, demonstrando que o volume de negociações $\left(V_{t}\right)$ é uma função logarítmica crescente do número de participantes $(N)$ e da força da nova informação disponível. Quanto maior a divergência entre as opiniões dos participantes (diferença entre otimistas e pessimistas diante da nova informação), menor a variação no volume transacionado.

A variação nos preços dos ativos vai depender do comportamento de cada participante perante a informação; alguns vão encarar as notícias com pessimismo, outros reagirão com otimismo. Além da força da nova notícia e de sua influência sobre o comportamento e a formação de expectativa individual do participante, a mudança de preços vai depender da oferta de ativos e, logicamente, do tamanho do mercado e do número de participantes. Assim sendo, o modelo prevê correlação positiva entre o valor absoluto da mudança de preços e o volume transacionado.

\section{Metodologia}

Nesta seção são descritos os procedimentos adotados na pesquisa para analisar a influência do mercado financeiro sobre o mercado futuro de commodities agrícolas. 


\subsubsection{Transmissão de volatilidade entre mercados}

O estudo do processo de transmissão de volatilidade entre os mercados financeiros, internacional e nacional, e o mercado futuro de contratos agropecuários no Brasil, está baseado na análise da volatilidade dos retornos dos preços 5 . O modelo considerado para essa análise é o modelo BEKK (Baba, Engle, Kraft \& Kroner), pertencente à classe dos modelos GARCH multivariados. Este modelo é utilizado por permitir a captação das interrelações dinâmicas entre as variâncias e covariâncias das séries financeiras, que são informações importantes, devendo ser consideradas no processo de seleção de portfólios de investimento. O modelo BEKK pode ser representado da seguinte forma:

$$
H_{t}=C^{\prime} C+\sum_{i=1}^{q} A_{i} \varepsilon_{t-1} \varepsilon_{t-1}^{\prime} A_{i}+\sum_{j=1}^{p} B_{j} H_{t-j} B_{j}
$$

em que: $H_{t}$ é a matriz de variâncias e covariâncias (VAR-COV) dos retornos do índice financeiro e do contrato futuro no tempo $t, C$ é uma matriz triangular inferior $K x K, A_{i}$ é uma matriz $K x K$ dos coeficientes do termo ARCH, $B_{j}$ é uma matriz $K x K$ dos coeficientes dos termos GARCH, $\varepsilon_{t}$ é um vetor de termos de erro que segue um processo M-GARCH, $k$ é o número de séries utilizadas e $q$ e $p$ são as ordens dos termos ARCH e GARCH, respectivamente ${ }^{6}$.

Nesta pesquisa, é estimado um modelo bivariado ${ }^{7}(\mathrm{~K}=2)$, com $\mathrm{p}=\mathrm{q}=1$, utilizando duas variáveis expressas sob a forma de taxas de retorno, sendo que o mercado financeiro internacional tem como proxy o índice acionário S\&P500 (DLOGSP50o) ${ }^{8}$, enquanto que o mercado financeiro nacional tem como proxy o índice Ibovespa (DLOGIND) 9 . O mercado de derivativos brasileiro é representado pelas séries de retornos referentes a quatro contratos futuros agropecuários negociados na BM\&FBOVESPA: boi gordo (DLOGBGI), café arábica (DLOGICF), milho ${ }^{10}$ (DLOGCCM) e soja (DLOGSFI). O número de

\footnotetext{
5 A taxa de retorno é expressa como: $R_{h}=\operatorname{Ln}\left(j, t^{I} / j-1^{I}\right)$

$6 \mathrm{Na}$ literatura pode-se encontrar a notação GARCH (p,q), ao invés de GARCH (q,p). Isto se deve à definição da ordem em que os termos aparecem na expressão. Nesta pesquisa, o software utilizado na estimação usa a notação (q,p).

7 A modelagem das séries financeiras é realizada aos pares, utilizando um índice financeiro e um contrato futuro de cada vez.

80 S\&P50o é um índice de avaliação das mudanças das condições do mercado acionário tendo como base o desempenho médio das 500 maiores empresas dos EUA. Autores como Harris (1982), Jegadeesh \& Subrahmanyam (1993), e Lopes (2006) utilizam esse índice em seus estudos como proxy para o mercado financeiro internacional.

9 IND é o código utilizado pela BM\&FBOVESPA para designar o índice Ibovespa. O Índice Bovespa é o mais importante indicador do desempenho médio das cotações do mercado acionário brasileiro. Sua relevância advém do fato do Ibovespa retratar o comportamento dos principais papéis (ações) negociados na BM\&FBOVESPA.

10 A BM\&FBOVESPA comercializa dois tipos de contrato futuro de milho, o contrato de base de preço de milho e o contrato de milho, que passou a ter liquidação financeira em 19/o9/2008. Nesta pesquisa, considerou-se apenas o antigo contrato com opção de entrega física e o atual contrato com liquidação financeira. As commodities agropecuárias açúcar cristal e etanol não puderam ser utilizadas devido à falta de informações referentes à comercialização em futuros para determinados períodos.
} 
parâmetros a serem estimados é 11.

Na análise da transmissão de volatilidade entre o mercado financeiro e o mercado de derivativos agropecuários, a significância individual dos coeficientes GARCH b $b_{12}$ indicará se parte da variância da série de retornos dos contratos futuros agropecuários, no período corrente, pode ser explicada pela variância do índice financeiro, no período anterior.

\subsubsection{Causalidade entre a volatilidade dos retornos dos índices financeiros e a volatilidade dos retornos do mercado futuro agropecuário no Brasil}

Para testar se a volatilidade dos retornos do índice S\&P5Oo e do Ibovespa causaram a volatilidade dos retornos dos contratos futuros de boi gordo, milho, café arábica e soja, no período 2002-2011, foi utilizado o teste de causalidade proposto por Cheung \& Ng (1996).

O primeiro estágio do teste envolve a estimação de modelos GARCH univariados para cada série de retornos, $X_{t}$ e $Y_{t}$ (retorno do índice financeiro e retorno do mercado futuro). Uma vez obtida as variâncias, por meio dos modelos GARCH, o segundo estágio consiste na construção da série dos quadrados dos resíduos padronizados proveniente dessa estimação. A correlação cruzada na defasagem (lag) $k, r_{u v}(k)$, é dada por:

$$
\operatorname{ruv}(k)=\operatorname{cuv}(k)(\operatorname{cuu}(0) \operatorname{cvv}(0))-\frac{1}{2}
$$

onde $C_{u v}(k)=T^{-1} \sum_{t=1}^{T}\left(U_{t}-\bar{U}\right)\left(V_{t}-\bar{V}\right) ; K=0, \pm 1, \pm 2, \ldots, c_{u u}(0)$ e $c_{v v}(0)$ são variâncias de $u$ e $v$, respectivamente, e $T$ é o tamanho da amostra.

O teste de Cheung \& Ng (1996), deste modo, é baseado na avaliação da significância da Função de Correlação Cruzada (FCC) dos resíduos padronizados ao quadrado $r_{u v}(k)$, sendo $u$ (correspondente à equação dos retornos do índice financeiro) e $v$ (correspondente à equação dos retornos do mercado futuro), visando detectar relações causais e identificar a direção da causalidade. Para o teste de causalidade em cada defasagem $k$, compara-se $\sqrt{T \hat{r}_{u v}(k)}$ com uma distribuição normal padronizada.

A partir dos resultados obtidos, é possível constatar se há evidências de que a volatilidade do mercado financeiro causa a volatilidade do mercado futuro agropecuário no Brasil, no período compreendido entre os anos de 2002 e 2011, visto que o incremento das negociações com derivativos agropecuários sugere que o fluxo de investimentos advindo do mercado financeiro internacional e nacional esteja atuando de forma a aumentar a volatilidade dos mercados futuros. 


\subsubsection{Relação entre volume negociado e retorno dos}

preços

Para detectar potencial relação causal entre o volume de contratos futuros negociados na BM\&FBOVESPA, no período 2002-2011, e os retornos das cotações desses contratos, utiliza-se análise baseada no modelo de Auto-Regressão Vetorial (VAR), cujas variáveis são o volume diário de negociação dos contratos futuros de, boi gordo (VOL_BGI), milho (VOL_CCM), café arábica (VOL_ICF) e soja (VOL_SFI) e os retornos das cotações futuras diárias desses contratos, boi gordo (DLOGBGI), milho (DLOGCCM), café arábica (DLOGICF) e soja (DLOGSFI). O modelo pode ser representado pelas seguintes equações:

$$
\begin{aligned}
& V_{t}=\alpha_{1}+\sum_{i=1}^{m} \beta_{1, i} V_{t-i}+\sum_{j=1}^{m} \gamma_{1, j} R_{t-j}+\epsilon_{1} \\
& R_{t}=\alpha_{2}+\sum_{i=1}^{m} \beta_{2, i} R_{t-1}+\sum_{j=1}^{m} \gamma_{2, j} V_{t-j}+\epsilon_{2}
\end{aligned}
$$

em que $V_{t}$ corresponde ao volume diário comercializado de cada contrato futuro agropecuário, $R_{t}$ corresponde ao retorno dos preços desses contratos, $\alpha, \beta$ e $\gamma$ são os parâmetros a serem estimados, $\epsilon_{1}$ e $\epsilon_{2}$ são as perturbações aleatórias não correlacionadas entre si e $m$ corresponde ao número de defasagens selecionadas segundo os critérios de Akaike e Schwarz.

Utilizando o método proposto por Granger (1969), as equações (3) e (4) permitem a identificação de relação linear causal entre volume e os retornos dos preços, que vai depender da significância estatística dos coeficientes estimados $\gamma_{1}$ da equação (3) e $\gamma_{2}$ da equação (4).

\subsection{Fonte de dados}

Utilizam-se nesta pesquisa dados referentes às cotações de ajuste ${ }^{11}$ do índice S\&P500, obtidas no site do YAHOO-Finanças, às cotações médias ${ }^{12}$ do índice Ibovespa ${ }^{13}$ e às cotações médias e volume transacionado dos contratos futuros de boi gordo, milho, café arábica e soja, ambos obtidos no site da BM\&FBOVESPA.

11 Cotação de ajuste é aquela referente ao último negócio registrado durante o call de fechamento, que ocorre nos últimos minutos de negociação do pregão diário. Foram utilizadas as cotações de ajuste do S\&P500 ao invés das cotações médias, porque as últimas não estavam disponíveis.

12 Cotação média é o preço médio do contrato registrado no decorrer do pregão diário.

13 Os retornos do Índice S\&P500 e do Ibovespa são calculados tendo como base sua cotação, apresentada como "ponto", que é uma medida de valor absoluto, cuja função é a de servir de instrumento de comparação, permitindo a análise de variação do valor de uma carteira de ativos ao longo do tempo. A rentabilidade, portanto, é representada pela variação dos pontos do índice. 
A princípio, a variável a ser utilizada para representar o mercado financeiro internacional seria o volume de capital estrangeiro entrante no mercado futuro agropecuário brasileiro, no entanto, como a mesma não está disponível, foi utilizado o índice da Standard and Poor's (S\&P50o), que tem sido adotado pela literatura, devido a sua importância, como proxy para o mercado financeiro internacional. A seleção do índice Ibovespa como variável representativa do mercado financeiro nacional seguiu o mesmo critério adotado para o S\&P5Oo e está baseada na sua importância como indicador do desempenho do mercado acionário brasileiro.

A periodicidade dos dados é diária, desconsiderando-se os dias em que não houve negociação nas bolsas nacional e internacional (sábados, domingos e feriados). Os dados referem-se ao período compreendido entre 02/01/2002 e 29/12/2011, com exceção do contrato futuro de soja, cuja série se inicia em 11/10/2002, já que A BM\&FBOVESPA não registrou negociação de contrato futuro de soja entre 13/06/2001 e 11/10/2002.

A seleção dos contratos baseou-se nos vencimentos com maior volume de negociação diária, ou seja, para cada dia de pregão foram considerados os contratos mais negociados, independente do mês de vencimento. Apesar de, geralmente, nas análises que contemplam os mercados futuros, a seleção dos contratos se basear nos primeiros vencimentos destes, nesta pesquisa deu-se prioridade à demanda diária pelos contratos, já que nem sempre os contratos para o primeiro vencimento foram os mais negociados do dia.

\section{Resultados e Discussão}

Nesta seção são apresentados e discutidos os resultados da pesquisa provenientes da aplicação dos modelos e procedimentos econométricos descritos anteriormente.

\subsection{Transmissão de volatilidade entre o mercado finan- ceiro internacional e o mercado futuro agropecuário no Brasil}

Na tabela 1 encontram-se os resultados da estimação do modelo BEKK ${ }^{14}$ (Equação 1) para os retornos do mercado financeiro internacional e os retornos do mercado futuro agropecuário brasileiro, no período 2002-2011 ${ }^{15}$. A significância estatística dos coeficientes GARCH indica que houve transmissão de volatilidade do mercado financeiro internacional para os mercados futuros de milho, café arábica e soja.

14 A ordem que se mostrou mais adequada para a estimação dos modelos foi o BEKK $(1,1)$, uma vez que as ordens superiores a estas não apresentaram coeficientes estatisticamente significantes.

15 Os resultados detalhados referentes às saídas do modelo BEKK estão disponíveis em Autor (2012). 
TABELA 1: RESULTADOS DO MODELO BEKK PARA OS RETORNOS DO ÍNDICE S\&P5O0 E DOS CONTRATOS FUTUROS, PERÍODO 2002-2011

\begin{tabular}{|c|c|c|c|c|}
\hline $\begin{array}{l}\text { Mercado } \\
\text { futuro }\end{array}$ & $\begin{array}{l}\text { Transmis- } \\
\text { são de vo- } \\
\text { latilidade }\end{array}$ & $\begin{array}{c}\mathbf{C} \text { o e f i - } \\
\text { cientes } \\
\text { G A R C H } \\
\left(\sigma_{t-1}^{2}\right) \\
\end{array}$ & Valor & p-value \\
\hline \multirow{2}{*}{ Boi gordo } & $\mathrm{MF} \rightarrow \mathrm{MFI}$ & $\mathrm{b}(2,1)$ & -0.002037 & $0.8169 \mathrm{NS}$ \\
\hline & $\mathrm{MFI} \rightarrow \mathrm{MF}$ & $b(1,2)$ & 0.003546 & $0.1162 \mathrm{NS}$ \\
\hline \multirow{2}{*}{ Milho } & $\mathrm{MF} \rightarrow \mathrm{MFI}$ & $b(2,1)$ & 0.005321 & $0.6563 \mathrm{NS}$ \\
\hline & $\mathrm{MFI} \rightarrow \mathrm{MF}$ & $\mathrm{b}(1,2)$ & -0.003092 & $0.0786 *$ \\
\hline \multirow{2}{*}{ Café arábica } & $\mathrm{MF} \rightarrow \mathrm{MFI}$ & $\mathrm{b}(2,1)$ & 0.020771 & $0.1763 \mathrm{NS}$ \\
\hline & $\mathrm{MFI} \rightarrow \mathrm{MF}$ & $\mathrm{b}(1,2)$ & -0.018528 & $0.0988 *$ \\
\hline \multirow{2}{*}{ Soja } & $\mathrm{MF} \rightarrow \mathrm{MFI}$ & $\mathrm{b}(2,1)$ & -0.011471 & $0.2853 \mathrm{NS}$ \\
\hline & $\mathrm{MFI} \rightarrow \mathrm{MF}$ & $\mathrm{b}(1,2)$ & 0.006999 & $0.0683 *$ \\
\hline
\end{tabular}

Fonte: Elaborada pelos autores com base nos resultados da pesquisa.

Notas: MF = Mercado Futuro, MFI = Mercado financeiro internacional.

*** denota significância estatística em nível de 1\%, ** denota significância estatística em nível de $5 \%$, *

denota significância estatística em nível de 10\%. NS = Indica estatisticamente não significante.

Em relação ao mercado futuro de boi gordo, a significância estatística dos coeficientes GARCH estimados apontam para a inexistência de transmissão direta de volatilidade, em qualquer direção. Entre os fatores que podem ter contribuído para estes resultados, as especificidades pertinentes à comercialização das commodities são relevantes.

No Brasil, o mercado futuro de boi gordo é caracterizado pelo baixo nível de participação dos investidores estrangeiros, principalmente em comparação aos investidores nacionais, motivo que pode estar associado ao fato de os contratos futuros de boi gordo, até o ano de 2011, permitirem o encerramento por entrega física, o que podia gerar para as partes do contrato (principalmente para o investidor internacional) problemas decorrentes dos altos custos de transação inerentes a este tipo de liquidação.

Por sua vez, a soja, o milho e o café arábica têm maior inserção no mercado internacional. Nos últimos anos, o milho é o derivativo agropecuário que vem apresentando o crescimento mais expressivo do número de negociações entre as commodities comercializadas na BM\&FBOVESPA, enquanto que $o$ mercado de café arábica é o que registra maior participação de investidores estrangeiros. A importância da soja também não pode ser desprezada, já que consiste em um dos principais produtos de exportação do país. 
Na Figura 1, encontram-se as volatilidades diárias estimadas a partir do modelo BEKK, para os retornos do mercado financeiro internacional e os retornos dos contratos futuros agropecuários, entre os anos de 2002 e 2011.

FIGURA 1: VOLATILIDADE DIÁRIA DOS RETORNOS DO ÍNDICE S\&P5OO E DOS CONTRATOS FUTUROS DE BOI GORDO, MILHO, CAFÉ ARÁBICA E SOJA, NO PERÍODO 2002-2011

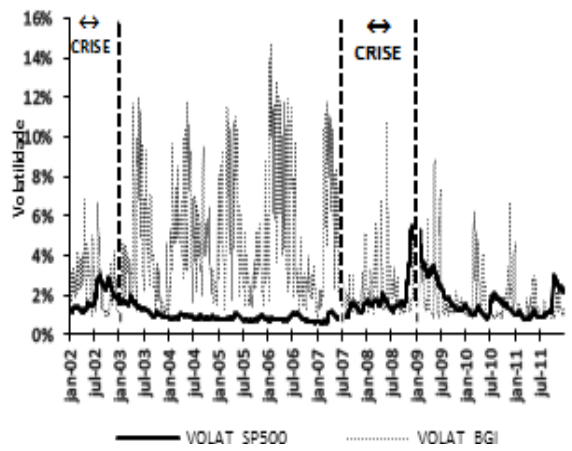

a) S\&P 500 - boi gordo

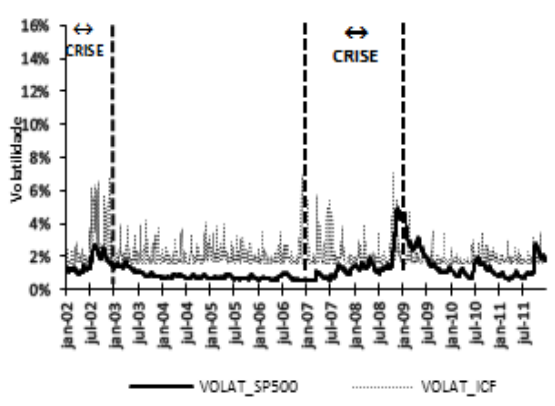

c) S\&P500 - café arábica

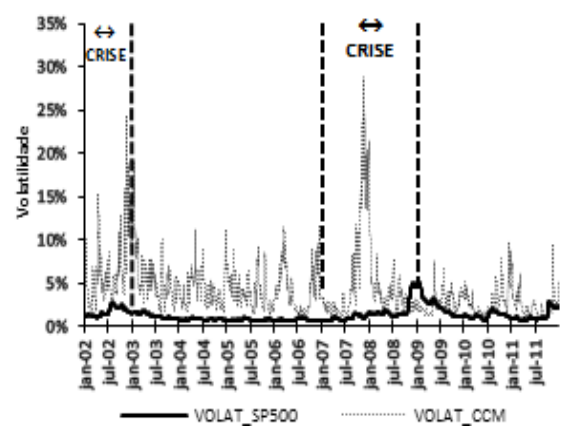

b) S\&P5Oo - milho

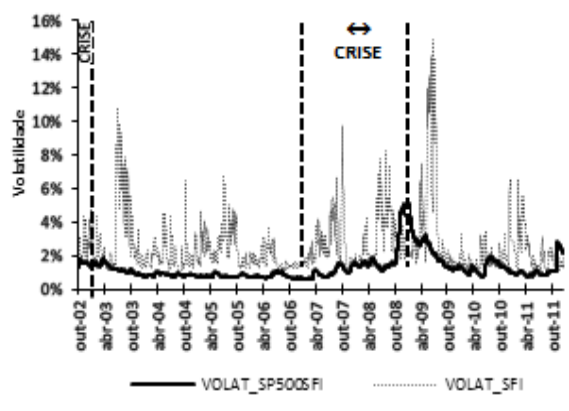

d) $\mathrm{S} \& \mathrm{P} 500-$ soja

Fonte: Elaborada pelos autores com base nos resultados da pesquisa.

Tanto no mercado financeiro internacional, quanto no mercado futuro de boi gordo, milho e café arábica, observa-se maior incidência de choques de volatilidade no ano de 2002, início do ciclo altista dos preços das commodities, e no biênio 2007-2008, período da crise financeira do subprime. No mercado futuro de soja, por sua vez, os maiores picos de volatilidade foram anotados nos anos de 2003 e 2009, refletindo certa demora de resposta do mercado de soja aos efeitos das crises internacionais.

Considerando todo o período analisado (2002-2011), apesar de durante a crise financeira do subprime (2007-2008) terem sido detectados alguns choques de volatilidade, as médias diárias não ficaram muito distantes das verificadas 
no período pré-crise. No pós-crise, há uma queda dos níveis de volatilidade, principalmente no mercado agropecuário, embora, a partir de 2010, já seja possível detectar o início de uma nova onda de inquietação, com a incidência de picos de volatilidade que, apesar de apresentarem menor magnitude dos que os verificados nos anos precedentes, podem estar refletindo os primeiros sinais da crise financeira europeia, iniciada na Grécia, em 2009.

A análise subsequente examinou a transmissão de volatilidade entre o mercado financeiro nacional, representado pelo índice acionário Ibovespa, e o mercado futuro agropecuário no Brasil. Os resultados encontram-se na Tabela 2.

TABELA 2: RESULTADOS DO MODELO BEKK PARA OS RETORNOS DO ÍNDICE IBOVESPA E DOS CONTRATOS FUTUROS, PERÍODO 2002-2011

\begin{tabular}{|c|c|c|c|c|}
\hline $\begin{array}{l}\text { Mercado } \\
\text { futuro }\end{array}$ & $\begin{array}{c}\text { Transmis- } \\
\text { são de vola- } \\
\text { tilidade }\end{array}$ & $\begin{array}{l}\text { Coeficien- } \\
\text { tes GAR- } \\
\text { CH }\left(\sigma_{t-1}^{2}\right) \\
\end{array}$ & Valor & p-value \\
\hline \multirow{2}{*}{ Boi Gordo } & $\mathrm{MF} \rightarrow \quad \mathrm{MFN}$ & $\mathrm{b}(2,1)$ & 0.0194531 & $0.0198 * *$ \\
\hline & $\mathrm{MFN} \rightarrow \mathrm{MF}$ & $\mathrm{b}(1,2)$ & -0.0015947 & $0.7373 \mathrm{NS}$ \\
\hline \multirow[t]{2}{*}{ Milho } & $\mathrm{MF} \rightarrow \quad \mathrm{MFN}$ & $\mathrm{b}(2,1)$ & 0.0033270 & $\begin{array}{c}0.7580 \\
\text { NS }\end{array}$ \\
\hline & $\mathrm{MFN} \rightarrow \mathrm{MF}$ & $\mathrm{b}(1,2)$ & 0.0044765 & 0.0794 * \\
\hline \multirow[t]{2}{*}{ Café arábica } & $\mathrm{MF} \rightarrow \quad \mathrm{MFN}$ & $\mathrm{b}(2,1)$ & -0.0206385 & $\begin{array}{c}0.2364 \\
\text { NS }\end{array}$ \\
\hline & $\mathrm{MFN} \rightarrow \mathrm{MF}$ & $\mathrm{b}(1,2)$ & 0.0223102 & $0.2125 \mathrm{NS}$ \\
\hline \multirow[b]{2}{*}{ Soja } & $\mathrm{MF} \rightarrow \mathrm{MFN}$ & $\mathrm{b}(2,1)$ & -0.0137923 & 0.1922 NS \\
\hline & $\mathrm{MFN} \rightarrow \mathrm{MF}$ & $\mathrm{b}(1,2)$ & -0.0032462 & $\begin{array}{c}\text { o. } 5674 \\
\text { NS }\end{array}$ \\
\hline
\end{tabular}

Fonte: Elaborada pelos autores com base nos resultados da pesquisa.

Notas: $\mathrm{MF}=$ Mercado Futuro, $\mathrm{MFN}=$ Mercado financeiro nacional.

*** denota significância estatística em nível de 1\%, ** denota significância estatística em nível de 5\%, * denota significância estatística em nível de 10\%. NS = Indica estatisticamente não significante.

A significância estatística dos coeficientes GARCH indica a ocorrência de transmissão de volatilidade do mercado financeiro nacional apenas para o mercado futuro de milho (ao nível de $5 \%$ ), enquanto que no mercado futuro de boi gordo, inesperadamente, a transmissão se dá na direção oposta, do mercado futuro para o mercado financeiro nacional (ao nível de 10\%). Não há indícios de transmissão de volatilidade entre o mercado financeiro nacional e os mercados futuros de café arábica e soja, em qualquer direção.

A ocorrência de transmissão de volatilidade do mercado futuro de boi gordo em direção ao mercado financeiro é, até certo ponto, surpreendente. Apesar 
de o mercado bovino ser representado no Ibovespa por empresas líderes do segmento de produção e processamento de proteína animal (JBS-Friboi e Marfrig), que juntas somam aproximadamente 1,8\% das ações que compõem esse índice, esta não é uma evidência forte o suficiente para justificar o resultado alcançado.

Considerando os resultados da análise, cabe ressaltar que a significância

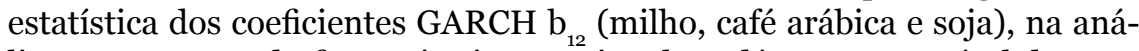
lise com o mercado financeiro internacional, se dá apenas ao nível de 10\%. Enquanto que, na análise com o mercado financeiro nacional, a significância estatística dos coeficientes $\mathrm{b}_{21}$ (boi gordo) e $\mathrm{b}_{12}$ (milho), se dão apenas ao nível de $5 \%$ e $10 \%$, respectivamente. Tal fato pode ser efeito das limitações da proxy utilizada, que abrange apenas o mercado acionário, não refletindo as atividades nos demais segmentos, como os mercados de títulos e derivativos financeiros, por exemplo.

Na Figura 2, são apresentadas as volatilidades diárias estimadas para as séries do Ibovespa (VOLAT_IND) e do mercado futuro de boi gordo (VOLAT_BGI), milho (VOLAT_CCM), café arábica (VOLAT_ICF) e soja (VOLAT_SFI).

Observa-se que os retornos do índice Ibovespa, assim como os retornos do S\&P500, apresentaram menor volatilidade diária do que os retornos dos contratos futuros agropecuários. A média diária de volatilidade do Ibovespa, no período 2002-2011, ficou em torno de 1,9\%, com o maior pico de volatilidade ocorrendo no segundo semestre de 2008 , auge da crise financeira do subprime, chegando a $5,5 \%$. 
FIGURA 2: VOLATILIDADE DIÁRIA DOS RETORNOS DO ÍNDICE IBOVESPA E DOS CONTRATOS FUTUROS DE BOI GORDO, MILHO, CAFÉ ARÁBICA E SOJA, NO PERÍODO 2002-2011
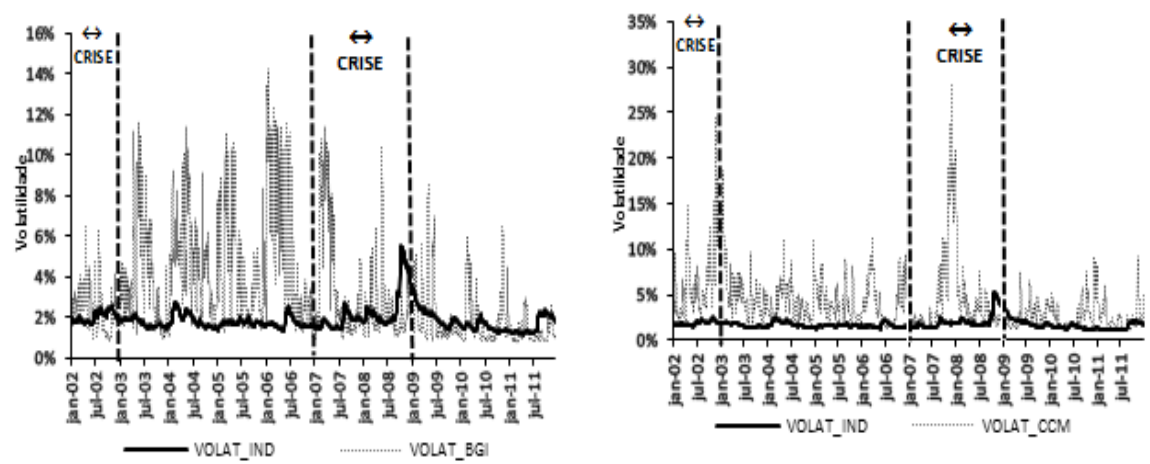

a) Ibovespa - boi gordo

b) Ibovespa - milho
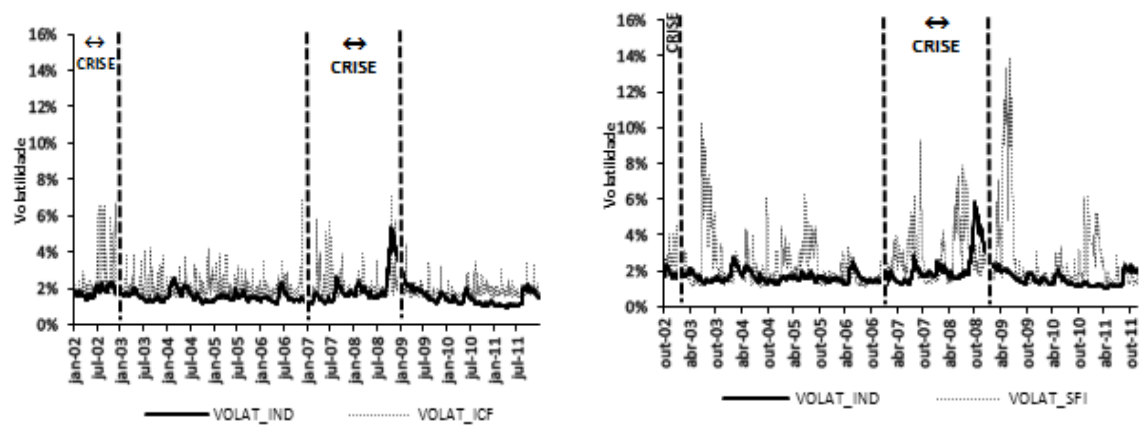

c) Ibovespa - café arábica

d) Ibovespa - soja

Fonte: Elaborada pelos autores com base nos resultados da pesquisa.

No pós-crise, a volatilidade diária do mercado financeiro nacional reduziu-se paulatinamente, chegando a níveis inferiores aos registrados no período précrise. Porém, a partir do segundo semestre de 2009, observou-se a ocorrência de pequenos choques, caracterizando comportamento semelhante ao verificado no mercado financeiro internacional e nos mercados futuros.

É possível verificar que, a partir da crise financeira do subprime, não foi verificado aumento significativo dos níveis de volatilidade, contrariando os pressupostos da teoria da financeirização, que defende que o mau desempenho dos ativos financeiros tradicionais, principalmente em tempos de crise, tem provocado uma migração de investimentos em direção aos mercados futuros, gerando uma bolha especulativa, responsável pela elevação da volatilidade dos preços nos mercados de alimentos. 


\subsection{Relação causal entre a variância dos mercados financeiros e a variância dos mercados futuros}

A análise realizada nesta seção utiliza o teste de causalidade proposto por Cheung \& Ng (1996) para verificar se a volatilidade dos retornos dos mercados financeiros, internacional e nacional, causou a volatilidade dos retornos dos contratos futuros agropecuários, no período 2002-2011.

As Tabelas 3 e 4 trazem, respectivamente, os resultados do teste de causalidade para o mercado financeiro, internacional e nacional, e os mercados futuros de boi gordo, milho, café arábica e soja.

TABELA 3: RESULTADOS DO TESTE DE CAUSALIDADE DE CHEUNG E NG (1996) PARA AS VOLATILIDADES DOS RETORNOS DO ÍNDICE S\&P5OO E DOS CONTRATOS FUTUROS DE BOI GORDO, MILHO, CAFÉ ARÁBICA E SOJA COMERCIALIZADOS NA BM\&FBOVESPA, PERÍODO 2002-2011

\begin{tabular}{l|l}
\hline Causalidade na variância & Resultados \\
\hline MFin $\rightarrow$ MFBGI & Não há causalidade \\
MFin $\rightarrow$ MFCCM & Não há causalidade \\
MFin $\rightarrow$ MFICF & Não há causalidade \\
MFin $\rightarrow$ MFSFI & Não há causalidade \\
MFBGI $\rightarrow$ MFin & Não há causalidade \\
MFCCM $\rightarrow$ MFin & Não há causalidade \\
MFICF $\rightarrow$ MFin & Não há causalidade \\
MFSFI $\rightarrow$ MFin & Não há causalidade \\
\hline
\end{tabular}

Fonte: Elaborada pelos autores com base nos resultados da pesquisa.

Notas: $\mathrm{H}_{\mathrm{o}}=$ não-causalidade da variância. MFin = Mercado financeiro internacional, MFBGI = Mercado futuro de boi gordo, MFCCM = Mercado futuro de milho, MFICF = Mercado futuro de café arábica, MFSFI = Mercado futuro de soja.

As estatísticas para o teste, baseadas na Função de Correlação Cruzada (FCC), com exceção da relação MFnac MFBGI (Tabela 4), não se mostram significativas, levando a concluir que não houve correlação entre os retornos em períodos diferentes. Desta forma, não se rejeita a hipótese de não-causalidade entre as variâncias dos retornos do mercado financeiro e dos retornos dos contratos futuros agropecuários. 
TABELA 4: RESULTADOS DO TESTE DE CAUSALIDADE DE CHEUNG E NG (1996) PARA AS VOLATILIDADES DOS RETORNOS DO ÍNDICE IBOVESPA E DOS CONTRATOS FUTUROS DE BOI GORDO, MILHO, CAFÉ ARÁBICA E SOJA COMERCIALIZADOS NA BM\&FBOVESPA, PERÍODO 2002-2011

\begin{tabular}{l|c}
\hline Causalidade na variância & Resultados \\
\hline MFnac $\rightarrow$ MFBGI & Há causalidade \\
MFnac $\rightarrow$ MFCCM & Não há causalidade \\
MFnac $\rightarrow$ MFICF & Não há causalidade \\
MFnac $\rightarrow$ MFSFI & Não há causalidade \\
MFBGI $\rightarrow$ MFnac & Não há causalidade \\
MFCCM $\rightarrow$ MFnac & Não há causalidade \\
MFICF $\rightarrow$ MFnac & Não há causalidade \\
MFSFI $\rightarrow$ MFnac & Não há causalidade \\
\hline
\end{tabular}

Fonte: Elaborada pelos autores com base nos resultados da pesquisa.

Notas: $\mathrm{H}_{\mathrm{o}}=$ não-causalidade da variância. $\mathrm{MFnac}=$ Mercado financeiro nacional, MFBGI = Mercado futuro de boi gordo, MFCCM = Mercado futuro de milho, MFICF = Mercado futuro de café arábica, MFSFI

= Mercado futuro de soja.

A causalidade demonstrada entre a variância do mercado financeiro nacional e a variância do mercado futuro de boi gordo (Tabela 4) nas defasagens $k=-4,-3$, indica que a variância dos retornos do Ibovespa, em $t_{-3}$ e $t_{-4}$, causaram a variância dos retornos do contrato futuro de boi gordo no período $t^{16}$. A detecção desta relação de causalidade, a princípio, é inusitada e de difícil justificação, uma vez que as empresas do setor pecuário, como já explicado, respondem por pequeno percentual do índice Ibovespa ${ }^{17}(1,8 \%)$.

Com exceção da relação MFnac MFBGI, os resultados obtidos se assemelham aos resultados alcançados por Galvão et al. (2000), que não encontraram relação causal entre as variâncias dos retornos diários do índice Ibovespa e a variância dos retornos diários dos contratos referenciados em índice Ibovespa. Maciel et al. (2010), que analisaram o impacto da variância dos contratos futuros de Ibovespa na variância dos índices Ibovespa, FGV-100, IBrX-50, IGC, SMLL, MLCX e Ibovespa Futuro também não puderem estabelecer relação causal entre a variância dos mesmos.

Por conseguinte, o caráter instabilizador atribuído aos mercados financeiros, apontado como principal fator gerador de incerteza aos participantes do mercado futuro agropecuário, não foi confirmado pelos resultados desta

16 Os resultados detalhados referentes a essa análise podem ser consultados em Autor (2012).

17 Essa é uma participação inexpressiva, principalmente comparada às empresas de outros segmentos, como as dos setores de petróleo e mineração que respondem, respectivamente, por 14,7\% e 14,5\% do total de ações que compõem o índice Ibovespa. 
pesquisa, o que não permite afirmar que a volatilidade dos retornos do mercado financeiro seja responsável pela volatilidade dos retornos do mercado futuro agropecuário no Brasil.

\subsection{Relação entre volume e variação dos preços futuros}

Os resultados provenientes da estimação do modelo VAR (Equações 3 e 4) para o volume de contratos futuros negociados e os retornos dos contratos futuros de boi gordo, milho, café arábica e soja podem ser visualizados na Tabela 5 .

Os dados indicam que não há relação causal partindo da variação do volume de contratos futuros negociados em direção aos retornos dos preços de nenhum dos contratos. No entanto, considerando a análise da relação causal partindo dos retornos dos contratos futuros em direção ao volume comercializado, os resultados foram divergentes.

Nos mercados futuros de boi gordo e soja não foi detectada relação causal, enquanto que nos mercados futuros de milho e café arábica ficou comprovado que os retornos dos preços futuros dos contratos precedem temporalmente o volume negociado. Deve-se considerar que esta análise não permite avaliar a existência de correlação contemporânea entre as variáveis ${ }^{18}$.

TABELA 5: RELAÇÃO LINEAR CAUSAL ENTRE VOLUME COMERCIALIZADO E RETORNOS DOS CONTRATOS FUTUROS AGROPECUÁRIOS, NO PERÍODO 2002-2011

\begin{tabular}{|c|c|c|c|c|}
\hline Análise & $\begin{array}{c}\text { Boi } \\
\text { gordo } \\
\text { (BGI) }\end{array}$ & $\begin{array}{l}\text { Milho } \\
\text { (CCM) }\end{array}$ & $\begin{array}{c}\text { Café ará- } \\
\text { bica (ICF) }\end{array}$ & $\begin{array}{c}\text { Soja } \\
\text { (SFI) }\end{array}$ \\
\hline $\begin{array}{l}\text { Relação linear } \\
\text { causal entre volume } \\
\text { comercializado e } \\
\text { retornos dos con- } \\
\text { tratos futuros }\end{array}$ & $\begin{array}{l}\text { Não há } \\
\text { relação }\end{array}$ & $\begin{array}{r}\text { DLOGCCM } \rightarrow \\
\text { VOL_CCM }\end{array}$ & $\begin{array}{r}\text { DLOGICF } \rightarrow \\
\text { VOL_ICF }\end{array}$ & $\begin{array}{l}\text { Não há } \\
\text { relação }\end{array}$ \\
\hline
\end{tabular}

Fonte: Elaborada pelos autores com base nos resultados da pesquisa.

Notas: DLOGCCM = retornos dos preços do contrato futuro de milho; DLOGICF = retornos dos preços do contrato futuro de café arábica; VOL_CCM = volume comercializado de contratos futuros de milho; VOL_ICF = volume comercializado de contratos futuros de café arábica.

As constatações provenientes desta análise estão de acordo com as apresentadas por outros trabalhos existentes na literatura, como os realizados por James \& Edmister (1983), Wood et. al. (1985) e Ajayi et al. (2009) ${ }^{19}$, por exemplo, estudaram a relação causal linear entre volume e preços em 
10 bolsas de valores localizadas na Europa ${ }^{20}$, constatando inexistência de relação causal entre volume e preço em quatro mercados (Holanda, Noruega, Portugal e Suíça), relação causal unidirecional em outros quatro mercados (Bélgica, Noruega, Espanha e Turquia) e relação causal bidirecional nas bolsas da Dinamarca e Grécia.

Com base nos resultados obtidos na presente pesquisa, não se pode afirmar que, em decorrência do aumento do volume de negociações com contratos futuros, verificado especialmente durante a crise financeira do subprime (2007-2008), tenha ocorrido aumento significativo da volatilidade dos preços futuros das commodities agropecuárias. Da mesma forma, não se pode concluir que a intensificação da comercialização de contratos futuros no Brasil esteja causando o aumento dos preços das commodities.

Com exceção da relação Ibovespa-Mercado Futuro de Boi Gordo, não há evidência de que a volatilidade dos mercados financeiros esteja causando a volatilidade dos mercados futuros de alimentos.

\section{Considerações Finais}

A análise da transmissão de volatilidade permite afirmar que, no período 2002-2011, houve transmissão de volatilidade do mercado financeiro internacional para o mercado futuro de milho, café arábica e soja. Com relação ao mercado financeiro nacional, os resultados apontam para transmissão de volatilidade deste mercado apenas para o mercado futuro de milho, enquanto que, no mercado futuro de boi gordo, a transmissão se dá na direção oposta, do mercado futuro para o mercado financeiro nacional. Não há indícios de transmissão de volatilidade entre o mercado financeiro internacional e o mercado futuro de boi gordo e o mercado financeiro nacional e os mercados futuros de café arábica e soja, em qualquer direção.

No entanto, convêm destacar que a significância estatística dos termos GARCH $b_{12}$ (milho, café arábica e soja), na análise com o mercado financeiro internacional, se dá apenas ao nível de 10\%. Enquanto que, na análise com o mercado financeiro nacional, a significância estatística dos coeficientes $\mathrm{b}_{21}(\mathrm{boi}$ gordo) e $b_{12}$ (milho), se dão apenas ao nível de $5 \%$ e 10\%, respectivamente. o que pode estar associado às limitações da proxy utilizada.

O teste de causalidade entre a variância dos retornos dos mercados financeiros, internacional e nacional, e a variância dos retornos dos mercados futuros indicou que, com exceção da relação Ibovespa-mercado futuro de boi gordo, não há evidências de causalidade entre a volatilidade dos retornos desses mercados, em nenhuma direção.

20 Os dados referentes aos preços e volume de transação das bolsas de valores analisadas por Ajayi et. al. são diários e referem-se ao período compreendido entre o4 de janeiro de 1982 e 12 de fevereiro de 1996. 
Os resultados também indicam não haver relação linear causal partindo da variação de volume de contratos futuros negociados em direção aos retornos dos preços. Na análise da relação causal partindo dos retornos dos preços dos contratos futuros em direção ao volume comercializado, os resultados indicam que nos mercados futuros de boi gordo e soja não há relação causal, enquanto que, nos mercados futuros de milho e café arábica, os retornos dos preços futuros precedem temporalmente o volume negociado. Deve-se considerar que esta análise não permite avaliar a existência de correlação contemporânea entre as variáveis.

Com base nesta pesquisa, não é possível confirmar a hipótese de que, no Brasil, no período 2002-2011, o aumento do volume de comercialização de contratos futuros esteja contribuindo para o aumento da volatilidade e ascendência dos preços das commodities agropecuárias. Contudo, deve-se considerar que esses resultados podem ser reflexos das características próprias atinentes ao mercado futuro agropecuário brasileiro, como o desenvolvimento ainda incipiente e o baixo volume de negociações, se comparado aos mercados internacionais, o que faz desse um mercado ainda pouco atraente aos investidores, principalmente aos estrangeiros.

\section{Referências}

Abanomey, Walid.S., Mathur, Ike (2001). "International portfolios with commodity futures and currency forward contracts." The Journal of Investing 10 (3): 61-68.

Ajayi, Richard A., Mehdian, Seyed., Mougoue, Mbodja (2009). "The Empirical Relation between Price Changes and Trading Volumes: Further Evidence from European Stock Markets." Alliance Journal of Business Research. URL[On line]: http://migre.me/b7Btr. Acesso em: 02 de julho de 2011.

Baffes, J., Haniotis, T. (2010). "Placing the 2006/2008 Commodity Price Boom into Perspective". Policy Research Working - Paper 5371: 1-40.

Bergstrom, G.L. (1975). "A New Route to Higher Returns and Lower Risk." The Journal of Portfolio Management 2: 30-38.

Bm\&Fbovespa - Mercadorias E Futuros - Boletim - "Resumo Estatístico do Pregão." URL[On line]: http://migre.me/ba3Ah. Acesso em 30 de outubro de 2011.

Bm\&Fbovespa - Mercadorias E Futuros. "Recuperação de Informações.” URL[On line]: http://www2.bmf.com.br/. Acesso em 30 de outubro de 2011.

Cheung, Y.W.; Ng, L.K. (1996). "A causality in variance test and its application to inancial market prices." Journal of Econometrics 72: 33-48.

Copeland, Thomas E. (1976). "A model of Asset Trading Under the Assumption of Sequential Information Arrival.” The Journal of Finance 31(4): 1148-168.

Domanski, Dietrich, Heath, Alexandra (2007). "Financial Investors and Commodity Markets.” Journal BIS Quartely Review: 53-67.

Erb, Claude B., Harvey, Campbell R. (2006). "The Tactical and Estrategic Value of Commodity Futures." Financial Analysts Journal 62 (2): 69-97. 
Galvão, Ana Beatriz C.; Portugal, Marcelo S.; Ribeiro, Eduardo P. (200o). "Volatilidade e Causalidade: Evidências para o Mercado à Vista e Futuro de Índice de Ações no Brasil." Revista Brasileira de Economia 54 (1): 37-56.

Gorton, Gary, Rowenhorst, K.G. (2006). "Facts and fantasies about commodity futures.” Financial Analysts Journal 62: 47-68.

Granger, C. W. J. (1969). "Investigating causal relations by econometric models and cross-spectral models.” Econometrica 37: 424-38.

Harris, L. (1982). A theoretical and empirical analysis of the distribution of speculative prices and of the relation between absolute price change and volume. Chicago: tese de doutorado, University of Chicago.

Irwin, Scott H.; Sanders, Dwight (2011). "Index Funds, Financialization, and Commodity Futures Markets.” Applied Economic Perspectives and Policy 33(1): 1-31.

James, C.; Edmister, R.O. (1983). “The Relation between Common Stock Returns Trading Activity and Market Value.” Journal of Finance 38: 1075-86.

Jegadeesh, Narasimhan; Subrahmanyam, Avanidhar (1993). "Liquidity Effects of the Introduction of the S\&P500 Index Futures Contract on the Underlying Stocks.” The Journal of Business 66(2): 171-87.

Lintner, J. (1965). "The valuation of risks assets: the selection of risk investments in stock portfolios and capital budgets." Review of Economics and Statistics 47 (1): 13-37.

Lopes, Daniel Costa (2006). Análise Quantitativa da Volatilidade entre os Índices Dow-Jones, Ibovespa e S\&P50o. Porto Alegre: dissertação de mestrado, Universidade Federal do Rio Grande do Sul, Programa de Pós-Graduação em Economia.

Maciel, Leandro dos Santos et al. (2010). "Impacto dos Contratos Futuros de Ibovespa na Volatilidade dos Índices de Ações no Brasil: Uma análise na Crise do Subprime”. URL[On line]: http://migre.me/b7BfQ. Acesso em 02 de setembro de 2011.

Markowitz, Harry (1952). "Portfolio Selection.” The Journal of Finance 7 (1): 77-91.

Masters, Michael W. (2008). "Testimony before the Committee on Homeland Security and Government Affairs.” URL[On line]: http://migre.me/b7BdS. Acesso em: 24 de setembro de 2012.

Mayer, Jörg (2009). "The Growing Interdependence Between Financial and Commodity Markets." UNCTAD/OSG/DP - Discussion Papers 195.

Smimou, Kamal (2010). "Stock Market and Agricultural Futures Diversification: An International Perspective.” The Journal of Alternative Investments 12(4): 36-57.

Sharp, Willian F. (1964). "Capital assets prices: a theory of market equilibrium under conditions of risk.” The Journal of Finance 19(3): 425-42.

Tang, Ke; Xiong, Wei (2010). "Index Investing and the Financialization of Commodities.” Working Paper, Department of Economics, Princeton University.

United Nations Conference On Trade And Development - Unctad (2009). "Trade and Development Report”. URL[On line]: http://www.unctad.org. Acesso em 12 de setembro de 2011.

Wood, R.A et al. (1985). "An investigation of Transactions Data for NYSE Stocks." Journal of Finance, 60: 723-39. 
SILVA, V. A.; MTTOS, L. B. Análise da Influência do Mercado Financeiro sobre o Mercado Futuro...

Yahoo Finanças. URL[On line]: http://br.finance.yahoo.com. Acesso em: 28 de novembro de 2011.

You, Leyuan, Daigler, Robert T. (2007). "Enhancing Diversification by Adding Commodity Futures.” URL[On line]: http://migre.me/b7B81. Acesso em 03 de abril de 2011. 